\title{
Objeto pedagógico virtual sobre Processo de Coordenação
}

\author{
Jocelyne da Cunha Bocchese ${ }^{1}$, Márcia Cristina Moraes², Valéria Pinheiro \\ Raymundo ${ }^{1}$ \\ ${ }^{1}$ Faculdade de Letras - Pontifícia Universidade Católica do Rio Grande do Sul (PUCRS) \\ Av. Ipiranga, 6.681, Porto Alegre, RS, Brasil \\ ${ }^{2}$ Faculdade de Informática - Pontifícia Universidade Católica do Rio Grande do Sul \\ (PUCRS) Av. Ipiranga, 6.681, Porto Alegre, RS, Brasil \\ Jocelyne@pucrs.br, MMoraesepucrs.br, Valepucrs.br
}

\begin{abstract}
The learning object Process of Coordination, whose steps of development and evaluation are presented in this paper, was elaborated in 2012 and integrates the repository of LAPREN (Learning Laboratory) of PUCRS. The material was elaborated in order to trigger the conscious processing system, considering the difficulties presented by freshmen and fostering autonomy in the learning process. Through questions and graded exercises, the learner is expected to build the concept of coordination and reflect about language use. The object was accessed 278 times so far and is related to other linguistic analysis materials of the set of LOs of Portuguese language.
\end{abstract}

Resumo. O objeto de aprendizagem Processo de Coordenação, cujas etapas de produção e de avaliação são aqui apresentadas, foi elaborado em 2012 e integra o acervo do Laboratório de Aprendizagem (LAPREN) da PUCRS. O material foi desenvolvido de modo a acionar o processamento do sistema consciente dos usuários, tendo em vista as dificuldades apresentadas por alunos ingressantes. Por meio de perguntas e atividades práticas, o aprendiz é levado a construir o conceito de coordenação e a aproximar a reflexão sobre a língua às possibilidades concretas de uso. O objeto, com 278 acessos até o momento, relaciona-se com outros materiais de análise linguística do acervo de objetos de língua portuguesa.

\section{Introdução}

O objeto de aprendizagem Processo de Coordenação: estudo dos elementos da organização estrutural da língua foi desenvolvido em 2012, integrando o repositório do Laboratório de Aprendizagem (LAPREN). O LAPREN, criado em 2009, é um ambiente de apoio à aprendizagem com vistas à ampliação e/ou reconstrução de conceitos. Nesse espaço, os alunos podem realizar estudos individualizados e/ou em grupo, utilizando os materiais pedagógicos produzidos por professores e bolsistas de Iniciação Científica. $\mathrm{O}$ acervo de objetos conta hoje com 80 materiais de diferentes áreas de conhecimento, dentre os quais 30 são de Língua Portuguesa. Com essa iniciativa, espera-se atender às 
necessidades específicas dos estudantes universitários e contribuir para a redução de desigualdades no acesso ao conhecimento.

Os objetos de aprendizagem (OAs) de língua portuguesa do LAPREN são concebidos a partir da percepção das principais dificuldades apresentadas por acadêmicos em seu desempenho linguístico, o que envolve, principalmente, leitura e domínio da escrita em diferentes situações comunicativas, especialmente quando é exigido o conhecimento da modalidade culta da língua. Entre as dificuldades apresentadas pelos estudantes - e esse diagnóstico é possível graças a dados provenientes de pesquisa em corpus constituído por redações de vestibular -, destacamse os problemas de estruturação frasal, talvez decorrentes da pouca familiaridade com a produção escrita de gêneros acadêmicos, os quais requerem atenção à construção de frases e compreensão dos princípios de ordenação dos constituintes, sejam eles termos ou orações.

Foi a partir da identificação dessas dificuldades que o objeto de aprendizagem "Processo de Coordenação: estudo dos elementos da organização estrutural da língua" foi desenvolvido. Neste artigo, inicialmente, apresentamos as bases teóricas e metodológicas que fundamentaram a construção desse material, enfocando o conceito de objeto de aprendizagem e de repositório de objetos, bem como os princípios linguísticos e pedagógicos referentes ao ensino da gramática e, mais especificamente, da relação sintática de coordenação; na sequência, apresentamos o processo de produção e de desenvolvimento de objetos do LAPREN, descrevendo as etapas de elaboração e de avaliação do objeto Processo de Coordenação, o qual faz parte do acervo da área de Letras.

\section{Bases teóricas e metodológicas}

Nesta seção, tratamos das peculiaridades dos objetos de aprendizagem e do repositório do LAPREN a partir de algumas definições existentes e dos princípios que orientam tanto a apresentação como a organização do conteúdo trabalhado no material.

\subsection{Objetos de aprendizagem e repositório de objetos}

Atualmente, não existe uma definição única e de consenso entre os pesquisadores da área do que sejam objetos de aprendizagem. De acordo com Downes (2004), existem várias definições, sendo algumas consideradas muito amplas e outras muito específicas. Sosteric e Hesemeir (2004) consideram que isso ocorre devido ao entendimento que os pesquisadores de diferentes áreas têm do conceito de objetos de aprendizagem.

De acordo com a IEEE, objetos de aprendizagem, ou OAs, podem ser entendidos como "qualquer entidade digital ou não digital que possa ser usada, reusada ou referenciada durante aprendizagem suportada pela tecnologia". Este é um exemplo de definição bastante ampla, pois qualquer material instrucional pode ser considerado um objeto de aprendizagem.

Para Sosteric e Hesemeier (2004), objetos de aprendizagem são arquivos digitais (imagens, filmes, etc) que podem ser usados para propósitos pedagógicos, incluindo, internamente ou por associação, sugestões sobre o contexto apropriado para uso do 
objeto. De acordo com os autores, esta definição limita o universo dos objetos de aprendizagem, seguindo o que é apontado pela literatura da área e prática atual.

Wiley (2000) e McGreal (2004) atribuem aos objetos de aprendizagem uma série de características: acessibilidade, considerando que, por estarem disponíveis na Internet, podem ser acessados de muitos locais; interoperabilidade, pois devem funcionar independentemente da plataforma de desenvolvimento (Windows, Linux, MacOS); adaptabilidade, já que seu uso pode ser adaptado a situações individuais de aprendizagem; reusabilidade, visto que podem ser utilizados em diferentes situações de aprendizagem; e recuperabilidade, visto que podem ser recuperados quando e onde for necessário.

A partir dos conceitos apresentados, levando em conta o contexto de desenvolvimento dos materiais, foi possível definir os OAs do LAPREN como materiais educacionais disponíveis em meio digital que permitem a interação do aluno com conteúdos específicos, acompanhados de exercícios autoexplicativos, com diferentes graus de dificuldade, para que o aluno possa monitorar seu aprendizado.

Para que os objetos de aprendizagem sejam disponibilizados à comunidade, é necessário que os mesmos sejam catalogados em repositórios. Um repositório é um local, normalmente integrado a um sistema de aprendizagem que organiza e armazena os materiais de modo a facilitar o acesso, permitindo sua utilização ou reuso em outras aplicações. Essa organização e armazenamento é realizada por meio de metadados, "dados sobre dados", que apresentam a descrição das características do material. É importante que os metadados sigam padrões que possibilitem a interoperabilidade entre os repositórios. Dentre os padrões existentes, podemos ressaltar o IEEE-LOM e Dublin Core.

Um exemplo de repositório digital é o DSpace, desenvolvido pelas bibliotecas do MIT (Massachusetts Institute of Technology) em conjunto com a HP (HewlettPackard), que tem como objetivo armazenar, preservar, gerir e disseminar o produto intelectual dos seus pesquisadores. O DSpace é disponibilizado livremente às instituições de pesquisa, sob a forma de um produto de código aberto, que pode ser adaptado e expandido funcionalmente (Smith, 2003). Por esse motivo, esse sistema foi escolhido para gerenciar o repositório de objetos de aprendizagem do LAPREN. O padrão de metadados utilizado pelo Dspace do LAPREN é o Dublin Core.

Atualmente, o referido repositório conta com 80 objetos de aprendizagem, sendo 33 da área de Matemática, 30 da área de Letras, 8 da área de Física, 6 da área de Química e 3 da área de Biologia. A diferença no número de objetos disponíveis para as áreas de Matemática e Letras ocorre devido ao fato de que estas áreas fazem parte do projeto do LAPREN desde sua inauguração. As áreas de Física e de Química foram incorporadas ao projeto no segundo semestre de 2011; e a área de Biologia, no segundo semestre de 2012.

Neste artigo, apresentamos o processo de desenvolvimento de um dos objetos de língua portuguesa: Processo de Coordenação. 


\subsection{Objeto de aprendizagem "Processo de Coordenação"}

Segundo Duarte (2007), o reconhecimento das relações de coordenação (e de subordinação) "é fundamental para que se tenha uma perfeita ideia da arquitetura do período". Entretanto, muitas gramáticas pedagógicas só abordam esse mecanismo no âmbito do período composto, quando, na verdade, palavras e termos simples podem aparecer coordenados entre si, desde que apresentem a mesma forma e exerçam a mesma função. De fato, conforme já definia Matoso Câmara (1971), "há uma composição lexical por coordenação (ex.: auriverde, luso-brasileiro) e a coordenação como construção sintática - a) de palavras, b) de grupos lexicais, c) de orações subordinadas a uma principal ou inteiramente independentes".

Em vista disso, no material elaborado procurou-se contemplar os diferentes níveis em que o mecanismo sintático da coordenação é utilizado em textos, o que, entende-se, pode contribuir para que as noções de equivalência e de independência sintática - que caracterizam as estruturas coordenadas - fiquem mais claras e abrangentes.

Com base na proposta de um ensino gramatical mais produtivo, fundamentado nos princípios da análise linguística, abordagem pedagógica que propõe uma compreensão mais ampla dos usos e do sistema linguístico do que a abordagem puramente gramatical, procurou-se conduzir a construção dos conceitos de maneira que o aprendiz pudesse não apenas identificar as estruturas coordenadas, mas também refletir sobre os fenômenos gramaticais e textuais que perpassam os usos da língua e os sentidos resultantes das escolhas feitas pelos falantes em uma determinada situação comunicativa, partindo de "uma reflexão explícita e organizada para resultar na construção progressiva de conhecimentos e categorias explicativas dos fenômenos em análise" (Mendonça, 2006).

Assim, a elaboração do objeto apoia-se na relação entre aprendizado e consciência. Para Baars (1988), é a consciência que permite a adaptação das informações novas ao contexto das informações já armazenadas na mente, provocando uma mudança nesse contexto e, consequentemente, promovendo o aprendizado. Em termos práticos, tal processo passa pela reflexão e pela compreensão de textos oferecidos à leitura e à análise.

Como o material foi desenvolvido para acionar o processamento do sistema consciente, o aluno é constantemente instigado a pensar, analisar e refletir sobre os itens linguísticos focalizados. Exige-se, sempre que possível, a verbalização escrita das conclusões que contribuem para a construção dos conceitos e dos princípios em análise. Espera-se que, ao fazê-lo, o aprendiz desenvolva capacidades e habilidades que lhe permitam melhorar seu desempenho linguístico e sua atenção executiva, com reflexos positivos no aproveitamento acadêmico. 


\section{Desenvolvimento do objeto "Processo de Coordenação"}

Nesta seção, apresentamos o contexto de produção, o desenvolvimento e a descrição do objeto "Processo de Coordenação.

\subsection{Produção e desenvolvimento de objetos de aprendizagem do LAPREN}

A produção e o desenvolvimento de objetos de aprendizagem do LAPREN envolve uma equipe multidisciplinar: professores que elaboram o objeto, professores que revisam a linguagem, professores que revisam a programação e a usabilidade, e programador Web.

O processo de produção e de desenvolvimento dos objetos de aprendizagem levam em conta as atividades de especificação, desenvolvimento, validação e evolução do software, previstas por Sommerville (2003), seguindo uma abordagem interativa e incremental (Larman, 2004). Assim, durante a fase de especificação, os professores responsáveis pelo conteúdo elaboram um storyboard inicial com o roteiro previsto para o objeto de aprendizagem. Após esse roteiro ser realizado, os professores se reúnem com a equipe de desenvolvimento (programador, professora da área de Comunicação, professora da área de Computação e professora responsável pela revisão linguística) para explicar o funcionamento do objeto, momento em que a equipe sugere aos docentes possibilidades de aperfeiçoamento de textos e de implementação do material: interação entre as telas, animações e exercícios; e questões de usabilidade do OA. Com a finalização do roteiro, inicia-se a fase de desenvolvimento, etapa em que o objeto de aprendizagem é programado e cadastrado no sistema LAPREN (Moraes, Raymundo, Bocchese e Lima, 2011).

Concluída a programação, o objeto é validado por meio de testes realizados tanto pela equipe de desenvolvimento quanto pelos elaboradores. Tendo passado com sucesso por essa avaliação, o objeto de aprendizagem é cadastrado no repositório do LAPREN e disponibilizado para acesso. Inicia-se, então, a fase de evolução, etapa em que o material passa a ser avaliado por bolsistas de Iniciação Científica vinculados ao laboratório. Caso seja necessário, o material pode sofrer atualizações.

Na sequência, apresentamos a descrição do objeto "Processo de Coordenação" e a ficha de avaliação utilizada no LAPREN pelos bolsistas.

\subsection{Descrição do objeto}

O objeto- Processo de Coordenação (Figura 1) - permite ao usuário compreender como se articulam os elementos coordenados em uma frase, sejam eles termos ou orações, de modo a construir indutivamente o conceito focalizado. A tela inicial, além de introduzir o assunto, sugerindo, através de imagens, o significado do conteúdo trabalhado, apresenta um menu que permite, a qualquer momento, o acesso e o retorno às diferentes etapas do estudo, retomando o conceito ou indo diretamente para os exercícios, entre outras possibilidades. O objeto apresenta, inicialmente, uma situação concreta - um texto sobre a Orquestra Sinfônica Brasileira (OSB) (Figuras 2 e 3) - para propor o problema inserido em um contexto comunicativo: como se organizam as frases e as ideias enumeradas em uma sequência? Por meio de perguntas condutoras, o aprendiz é levado a observar e analisar estruturas coordenadas, comparando-as, manipulando-as, 
II Congresso Brasileiro de Informática na Educação (CBIE 2013)

XIX Workshop de Informática na Escola (WIE 2013)

construindo hipóteses e formulando conclusões parciais que o levem à construção do conceito de coordenação.

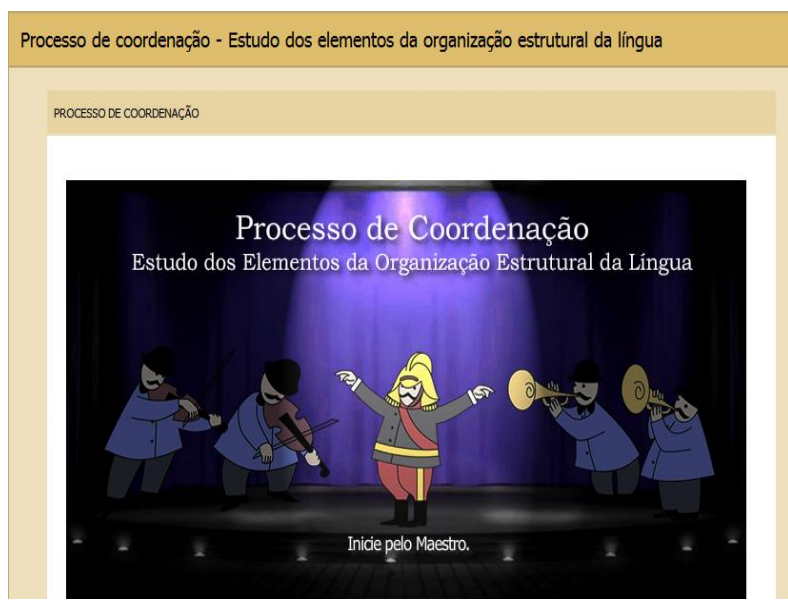

Figura 1. OA Processo de Coordenação

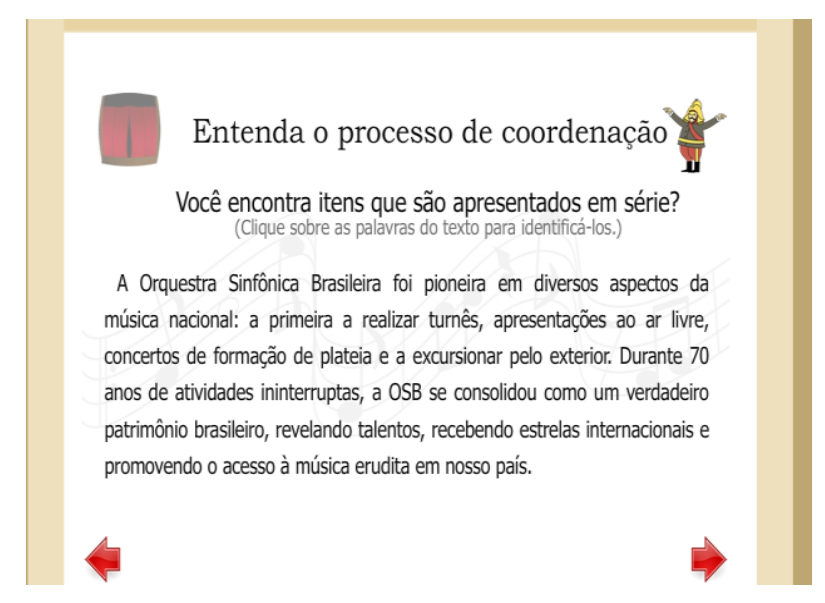

Figura 2. OA Processo de Coordenação: texto sobre a OSB

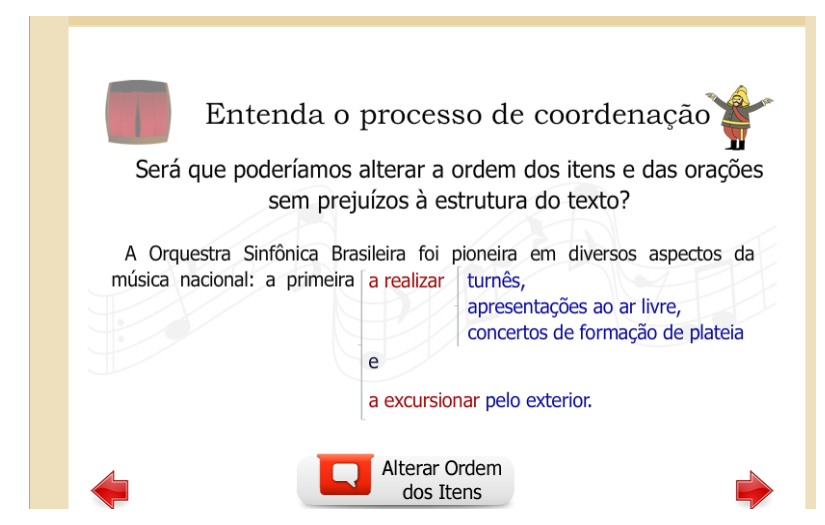

Figura 3. OA Processo de Coordenação: elementos coordenados 
$\mathrm{Na}$ sequência, são propostas atividades práticas sob a forma de exercícios gradativos, que focalizam, inicialmente, a identificação e a transformação das estruturas em estudo, para, num estágio mais avançado, promoverem a produção de um parágrafo (OA Processo de Coodenação - Figuras 4 e 5). Além da nota informativa sobre a OSB, o OA aqui apresentado propõe a leitura e a análise de diferentes textos, tais como uma definição (de "musicoterapia"). Procura-se, assim, contemplar a exposição do aprendiz à diversidade de gêneros, embora os textos selecionados sejam, necessariamente, de curta extensão para que leitura se complete em uma única tela do monitor.

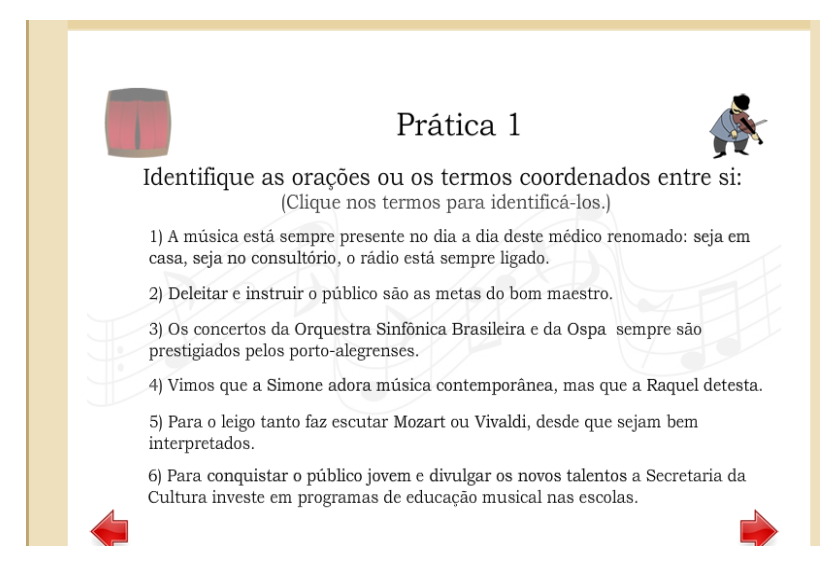

Figura 4. OA Processo de Coordenação: exercício de identificação

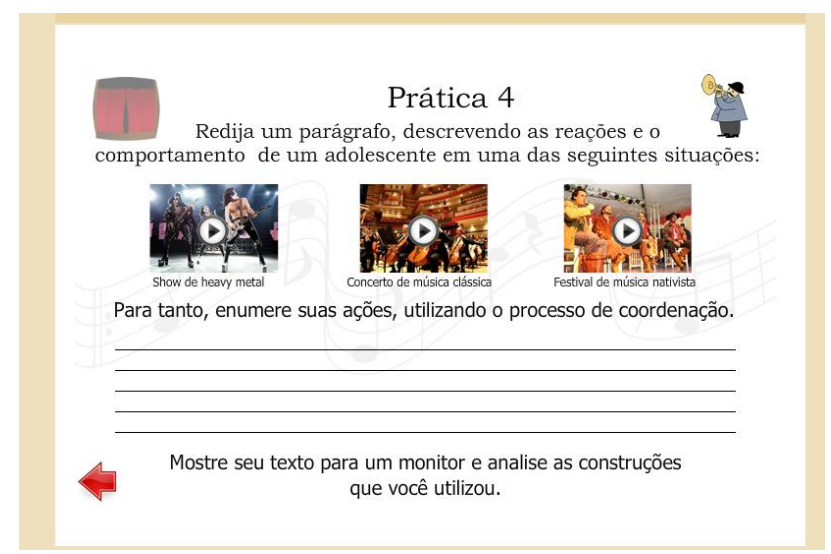

Figura 5. OA Processo de Coordenação: produção de um parágrafo

Visando promover a autonomia no processo de aprendizagem, todas as perguntas dirigidas aos alunos, assim como os exercícios propostos, permitem o acesso à(s) resposta(s) possível(eis). Para avaliar e corrigir suas respostas, comparando-as com as sugeridas pelo programa, o usuário pode teclar o comando Conferir a Resposta ou Resposta Sugerida na parte inferior da tela. Algumas respostas - especialmente as que envolvem práticas de análise de textos ou de reescrita de períodos - são acompanhadas de explicações complementares que orientam a análise, solicitam a refacção do exercício e justificam os acertos. Assim, o conteúdo vai sendo retomado e reforçado, num ir e vir que busca aproximar a reflexão sobre a língua às possibilidades concretas de uso. Quanto à Prática (ver Figura 5), cuja resposta é aberta, pretende-se, com ela, incentivar a socialização da escrita, inerente à natureza sociointerativa da linguagem. 
Mesmo que o usuário acesse o objeto em outros ambientes que não o laboratório ou a sala de aula, poderá copiar e reler o(s) seu texto(s) mais tarde, mostrando-o(s) para pessoas que possam apreciá-lo.

\subsection{Ficha de avaliação}

Com a disponibilização do objeto no repositório institucional, inicia-se a etapa de avaliação realizada por bolsistas de Iniciação Científica da área de Letras. O instrumento utilizado para avaliar o objeto é uma Ficha de Avaliação, que, por meio de perguntas, permite ao avaliador analisar quatro diferentes aspectos do material, dando-lhes a possibilidade de fazer comentários e sugerir correções, justificando-as. Segue, abaixo, os itens que compõem a Ficha de Avaliação utilizada para análise dos objetos do LAPREN (Tabela 1).

Tabela 1. Ficha de avaliação

\begin{tabular}{|c|c|c|c|}
\hline 1.CADASTRO & Sim & Em parte & Não *Justifique \\
\hline 1.1 O cadastro está completo? & $\mathrm{O}$ & $\mathrm{O}$ & $\mathrm{O}$ \\
\hline $1.2 \mathrm{O}$ cadastro está adequado? & $\mathrm{O}$ & $\mathrm{O}$ & $\mathrm{O}$ \\
\hline $1.3 \mathrm{O}$ cadastro está coerente com o conteúdo abordado? & $\mathrm{O}$ & $\mathrm{O}$ & $\mathrm{O}$ \\
\hline
\end{tabular}

\begin{tabular}{|c|c|c|c|}
\hline 2.1 As explicações estão claras e completas? & $\mathrm{O}$ & $\mathrm{O}$ & $\mathrm{O}$ \\
\hline 2.2 As explicações auxiliam a construir o conceito? & $\mathrm{O}$ & $\mathrm{O}$ & $\mathrm{O}$ \\
\hline 2.3 Os exemplos são elucidativos? & $\mathrm{O}$ & $\mathrm{O}$ & $\mathrm{O}$ \\
\hline 2.4 O conteúdo está organizado de forma progressiva? & $\mathrm{O}$ & $\mathrm{O}$ & $\mathrm{O}$ \\
\hline $2.5 \mathrm{O}$ aluno é instigado a refletir sobre o conteúdo? & $\mathrm{O}$ & $\mathrm{O}$ & $\mathrm{O}$ \\
\hline 2.6 Há quantidade suficiente de atividades práticas? & $\mathrm{O}$ & $\mathrm{O}$ & $\mathrm{O}$ \\
\hline
\end{tabular}

\begin{tabular}{|c|c|c|c|}
\hline $3.1 \mathrm{O}$ tamanho da letra é adequado? & $\mathrm{O}$ & $\mathrm{O}$ & $\mathrm{O}$ \\
\hline $3.2 \mathrm{O}$ esquema de cores contribui para a legibilidade? & $\mathrm{O}$ & $\mathrm{O}$ & $\mathrm{O}$ \\
\hline 3.3 O espaçamento entre linhas e parágrafos é adequado? & $\mathrm{O}$ & $\mathrm{O}$ & $\mathrm{O}$ \\
\hline
\end{tabular}

\section{NAVEGAÇÃO}

\begin{tabular}{|c|c|c|c|}
\hline 4.1 A navegação permite acesso às diferentes etapas do objeto? & $\mathrm{O}$ & $\mathrm{O}$ & $\mathrm{O}$ \\
\hline 4.2 A navegação permite autonomia nos exercícios? & $\mathrm{O}$ & $\mathrm{O}$ & $\mathrm{O}$ \\
\hline 4.3 Os marcadores de navegação fornecem indicações claras? & $\mathrm{O}$ & $\mathrm{O}$ & $\mathrm{O}$ \\
\hline 4.4 Há possibilidade de refazer/corrigir os exercícios? & $\mathrm{O}$ & $\mathrm{O}$ & $\mathrm{O}$ \\
\hline
\end{tabular}

\footnotetext{
Comentários e sugestões:
} 
Há correções a serem feitas? ( ) Sim $\quad(\quad) \quad$ Não $\quad$ Qual/Quais?

*Justificativa

Com 278 acessos, desde que foi disponibilizado, o material foi avaliado pelos elaboradores, pela equipe de desenvolvimento e por quatro bolsistas de Iniciação Científica. A equipe de desenvolvimento, constituída por professores das áreas de informática e de comunicação, avalia o material ao longo do processo de criação, sugerindo alterações no layout e testando as condições de usabilidade. No caso específico deste objeto, foram sugeridas apenas duas alterações: a redução dos textos iniciais e o aumento da fonte, para adequação ao tamanho da tela. Quanto aos bolsistas de Iniciação Científica, não foram identificados problemas quanto ao conteúdo e à usabilidade; houve apenas comentário sobre o tempo de execução de todas as práticas, com sugestão de que houvesse um registro, no próprio sistema, das etapas vencidas a cada acesso e do retorno do mesmo usuário ao OA. Para implementar esse tipo de registro, estuda-se a viabilidade de anexar, aos objetos do repositório, algum tipo de dispositivo a partir do qual o usuário possa assinalar o momento em que interrompeu o estudo, as etapas retomadas e o momento da conclusão, de modo que seja possível, para a equipe do LAPREN, recuperar o histórico de acessos de cada objeto pelo mesmo usuário.

No momento, o objeto está inserido em uma área moodle, para ser avaliado por professores da educação básica em oficinas previstas para o final de 2013. A proposta das oficinas é incentivar os professores a utilizarem o objeto como material de ensino e aprendizagem, qualificando a prática docente. Além disso, com esse trabalho, pretendese que o conteúdo do objeto seja validado por mais professores de língua portuguesa, os quais deverão preencher a ficha de avaliação apresentada neste artigo.

\section{Considerações finais}

O OA "Processo de Coordenação" foi desenvolvido para acionar o processamento do sistema consciente, instigando o usuário a pensar, analisar e refletir sobre os conceitos e os itens linguísticos focalizados. Em termos práticos, o objeto pode ser utilizado pelo professor para introduzir o conceito de coordenação ou para complementar as explicações em sala de aula. O material pode também ser utilizado pelo aluno para aprender, revisar e praticar o tópico abordado, de forma autônoma.

No primeiro caso, o professor pode projetar as telas de modo pausado, solicitando a participação individual dos alunos nas respostas às questões condutoras, comparando e analisando essas contribuições antes de apresentar as definições fornecidas pelo programa. No segundo caso, o aluno pode trabalhar individualmente ou com um colega, seguindo a sequência sugerida pelo material ou acessando a etapa que desejar. Pode, ainda, interagir com todo o objeto ou com parte dele.

Por fim, é importante informar que o OA aqui apresentado encontra-se, ainda, em fase de validação e relaciona-se com outros objetos de análise linguística do acervo institucional, tais como o que trata do paralelismo sintático e semântico, os cinco OAs que abordam o emprego da vírgula, os três que tratam do emprego do pronome relativo 
"que" e os que analisam os mecanismos responsáveis pela tessitura textual (Tecendo Textos I e II - este último em fase de programação).

Cumpre lembrar, também, que, embora constituam um importante auxílio à aprendizagem, os objetos - mesmo quando reunidos em um vasto repositório - não substituem o estudo sistemático e aprofundado da língua, o que envolve muita leitura, muita escrita, consulta a gramáticas, a dicionários e a outras fontes teóricas consistentes.

\section{Referências}

Baars, B.(1988) "A Cognitive theory of consciousness". New York: Cambridge University Press.

Downes, S. (2004) "Learning objects: resources for learning worldwide in Online Education using Learning Objects", editado por R. McGreal. New York: RoutledgeFalmer, p. 21-31.

Duarte, M. E. (2007) “Coordenação e subordinação”. IN: BRANDÃO, Silvia Figueiredo \& VIEIRA, Silvia Rodrigues. Ensino da gramática: descrição e uso. São Paulo: Contexto.

Dublin Core. "Dublin Core Metadata Initiative", http://dublincore.org/, July.

IEEE. "IEEE Learning Object Metadata", http://ltsc.ieee.org/wg12/, July.

Larman, C. (2004) “Agile and Interative Development: A Manager's Guide”. Boston: Addison-Wesley.

Mattoso Câmara, J. (1971) “Dicionário de Filologia e Gramática”. Rio de Janeiro: Jozon Editor.

McGreal, R. (2004) "Introduction in Online Education using Learning Objects”, editado por R. McGreal. New York: RoutledgeFalmer, p. 1-16.

Mendonça, M. (2006) "Análise linguística no ensino médio: um novo olhar, um outro objeto”. IN: Bunzen, Clécio \& Mendonça, Márcia (orgs.) Português no ensino médio e formação do professor. São Paulo: Parábola Editorial.

Moraes, M. and Raymundo, V. and Bocchese, J. and Lima, V. "Elaboração de Objetos de Aprendizagem para o LAPREN: Processo de Desenvolvimento e Sistema de Produção", http://www.br-ie.org/sbie-wie2011/, June.

Smith, M. et al. (2003) "Dspace: an open source dynamic digital repository". D-Lib Magazine, 9, 1, http://www.dlib.org/dlib/january03/smith/01 smith.html,July.

Sommerville, I. (2003) "Engenharia de software”. São Paulo: Pearson Education.

Sosteric, M. and Hesemeier, S. (2004) "A first step towards to a theory of learning objects" in Online Education using Learning Objects, editado por R. McGreal. New York: RoutledgeFalmer, p. 32-42.

Wiley, D. (2000) "Connecting learning objects to instructional design theory: a definition, a metaphor and a taxonomy". In: The instructional use of learning objects - On-line Version, http://reusability.org/read/, June. 